\title{
Stone-Wales transformations triggered by intrinsic localized modes in carbon nanotubes
}

\section{$\operatorname{AUTHOR}(\mathrm{S})$ :}

Shimada, Takahiro; Shirasaki, Daisuke; Kitamura, Takayuki

\section{CITATION:}

Shimada, Takahiro ... [et al]. Stone-Wales transformations triggered by intrinsic localized modes in carbon nanotubes. PHYSICAL REVIEW B 2010, 81(3): 035401.

ISSUE DATE:

2010

URL:

http://hdl.handle.net/2433/148378

RIGHT:

(C) 2010 The American Physical Society 
PHYSICAL REVIEW B 81, 035401 (2010)

\title{
Stone-Wales transformations triggered by intrinsic localized modes in carbon nanotubes
}

\author{
Takahiro Shimada, * Daisuke Shirasaki, and Takayuki Kitamura \\ Department of Mechanical Engineering and Science, Kyoto University, Sakyo-ku, Kyoto 606-8501, Japan \\ (Received 25 June 2009; revised manuscript received 22 November 2009; published 5 January 2010)
}

\begin{abstract}
The crucial role of intrinsic localized modes (ILMs) in the atomic scale as a trigger of defect nucleation was studied using molecular-dynamics simulations for a $(5,5)$ armchair carbon nanotube (CNT) under axial tension. A localized vibration at a pair of neighboring atoms was found to be the ILM, which simultaneously produces an intense concentration of kinetic energy, even in the structurally homogeneous CNT. The excited ILM was gradually amplified by the nonlinearity of C-C interaction. The amplified ILM, then, drove the breaking of the on-site C-C bond, which leads to the Stone-Wales transformation producing a topological defect consisting of two pentagons and two heptagons coupled in pairs. This signifies that mechanical instability can be activated by the ILMs. Such mechanism is expected to apply to other mechanical instabilities, e.g., as an origin of phase transformations in silicon under hydrostatic pressure.
\end{abstract}

DOI: $10.1103 /$ PhysRevB.81.035401

PACS number(s): 05.45.Yv, 62.25.- g, 63.22. $-\mathrm{m}$

\section{INTRODUCTION}

Since the discovery of intrinsic localized mode (ILM) or discrete breather by Takeno and co-workers, ${ }^{1,2}$ the fundamental properties of ILMs have been theoretically investigated in an ideal one-dimensional or two-dimensional (2D) lattice using numerical simulations with imaginary potentials. ${ }^{3-8}$ The ILM is a time-periodic and spatially localized vibration that characteristically appears in a lattice system consisting of discrete elements with nonlinear interaction. During the excitation of ILMs, the lattice vibration is locally amplified and the frequency is enhanced by the nonlinearity of interaction beyond the upper bound of the phonon-dispersion relation. Moreover, the amplified vibration of ILMs gives rise to a concentration of kinetic energy at the local excitation site, even in a homogeneous lattice system. In recent years, the ILM has been observed experimentally in various physical contexts. ${ }^{9-13}$ Thus, the ILM research is expected to expand further in various fields.

An atomic system, which possesses both the discreteness of atomic arrangement and the nonlinearity of interatomic interaction, is one of the new fields exploited to the ILMs. The existence of ILMs in the atomic system was first demonstrated by Yamayose et al. ${ }^{14}$ using molecular-dynamics (MD) simulations for a 2D graphite sheet with a realistic interatomic potential. The ILM excitation was studied extendedly in a quasi-three-dimensional atomic component of a carbon nanotube (CNT) ${ }^{15,16}$ which is made by rolling up a graphite sheet. These atomic-level studies mainly focused on the excitation and fundamental properties of the ILM itself. However, one may have a simple but practical question, "what does the excited ILM act as in the atomic components?" in other words, the crucial role of ILMs in the atomic scale, which has never yet been discussed.

Materials strength of atomic components is basically predominated by mechanical instability, which is characterized by a bond switching. ${ }^{17}$ In general, the mechanical instability occurs preferentially at a local site where stress or strain concentrates due to structural inhomogeneity, for example, dislocation emissions from a crack tip. As for mechanical instability in the CNTs, a topological defect that consists of two pentagons and two heptagons coupled in pairs (5-7-7-5) appears by the $\mathrm{C}-\mathrm{C}$ bond rotation at a local site of the armchair CNTs under axial tension (the so-called Stone-Wales transformation $\left.{ }^{18}\right)$. The formation of a (5-7-7-5) defect plays a central role in determining the ductile behavior of CNTs because the defect acts as a source of the (5-7) and (7-5) dislocations. ${ }^{19,20}$ In contrast to inhomogeneous atomic components, temporal localization of kinetic energy is essential for the initiation of the Stone-Wales transformation because there is no stress or strain concentration in the defect-free CNTs due to their homogeneous atomic structure. Remembering that the ILM gives rise to the intensive energy concentration even in the absence of structural inhomogeneity by its localized vibration with a large amplitude, there is a possibility that the excited ILM in the armchair CNT could trigger the Stone-Wales transformation.

In this paper, we carry out MD simulations for the $(5,5)$ armchair CNT under axial tension with the aim to elucidate the crucial role of the excited ILMs in the atomic scale as a trigger of the Stone-Wales transformation.

\section{SIMULATION PROCEDURE}

Figure 1(a) shows the simulation model of the $(5,5)$ armchair CNT consisting of 280 carbon atoms. The TersoffBrenner potential ${ }^{21-23}$ is employed to describe the interaction of carbon atoms. The initial (unstrained) diameter and axial length of the model were set to $d_{0}=6.96 \AA$ and $L_{0}$ $=35.27 \AA$, respectively. The periodic boundary condition was applied in the axial $z$ direction. Note that the periodic boundary condition does not affect the results (e.g., the number of ILMs excited per unit length and lifetime of ILMs) with the simulation cell length in the $z$ longer than $30 \AA . .^{15}$ Hence, there is no influence of the periodic boundary condition as well as the finite-size effect.

The axial tensile strain of $\varepsilon_{z z}=0.10$ was applied to the $(5,5) \mathrm{CNT}$. Then, the atomic configuration was fully relaxed using the fast inertial relaxation engine algorithm ${ }^{24}$ until the force acting on each atom was less than $1.0 \times 10^{-8} \mathrm{nN}$. Then, the diameter and axial length of the CNT under ten- 


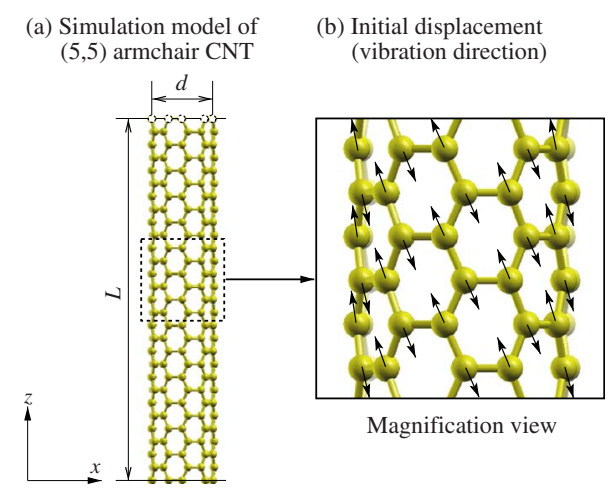

FIG. 1. (Color online) (a) Simulation model of the (5,5) armchair CNT. (b) Initial displacement (vibration direction). Arrows indicate the direction of initial displacement. The angle of atomic vibration with respect to the tube axis is about $25^{\circ}$.

sion were preliminarily calculated to be $d=6.73 \AA$ and $L$ $=38.80 \AA$, respectively.

The ILM is, in general, excited from the modulational instability of the phonon mode having the maximum angular frequency (zone-boundary mode). ${ }^{25}$ Since the ILMs were successfully generated in a graphite sheet ${ }^{14}$ by applying a displacement of the zone-boundary phonon mode and a minute disturbance of small momentum, we followed the same procedure here. As an initial condition, we applied an atomic displacement with the same amplitude of $0.085 \AA$ that corresponds to zone-boundary phonon mode of the strained CNT and momentum equal to $10 \mathrm{~K}$ for a minute disturbance in order to induce the modulational instability of the applied zone-boundary mode. The orientation of the initial displacement (vibration direction) is shown in Fig. 1(b). The angle of the vibration direction with respect to the tube axis was about $25^{\circ}$.

The microcanonical MD simulations were carried out for the $(5,5)$ CNT with the above-mentioned initial condition under tensile strain of $\varepsilon_{z z}=0.10$. The Verlet's algorithm ${ }^{26}$ was employed for the numerical integration with a sufficiently small time step of $0.01 \mathrm{fs}$ to exclude non-negligible truncation error that would otherwise result in difficulty in analyzing the atomic vibration.

\section{RESULTS AND DISCUSSION}

The potential energy of the $i$ th atom, $E_{i}^{\text {pot }}$, can be calculated within the framework of the Tersoff-Brenner potential. The kinetic energy of the $i$ th atom, $E_{i}^{\mathrm{kin}}$, can be calculated from its momentum as well. Hence, the total energy of the $i$ th atom, $E_{i}$, can be obtained by a simple summation of the two terms, $E_{i}=E_{i}^{\text {pot }}+E_{i}^{\text {kin }}$.

Figure 2 plots the change in the maximum total energy of an atom, $E_{\max }=\max \left(E_{i}\right)$, in each time step during the MD simulation. At the beginning of the simulation, $E_{\max }$ started to increase rapidly and then maintained a relatively high energy state of $1.3-2.3 \mathrm{eV}$ from 0.1 to 0.4 ps. Such a dramatic increase in the maximum energy of an atom has been characteristically observed when the ILM was excited in a graphite sheet or CNT. ${ }^{14-16}$ In these previous studies, $E_{\max }$ finally

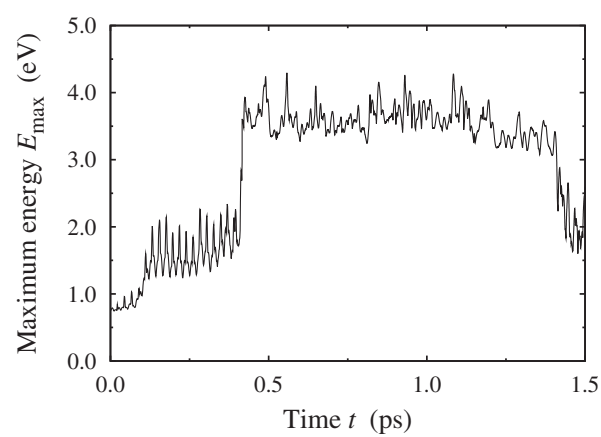

FIG. 2. Change in maximum energy of an atom, $E_{\max }$, during the simulation.

decreased the moment the excited ILM disappeared. On the other hand, a further increase in $E_{\max }$ was found in this study after 0.4 ps to a higher energy state of 3.5-4.0 eV. Below, we discuss separately what occurred in the $(5,5)$ CNTs during (i) $0.1-0.4 \mathrm{ps}$ (the medium energy state) and (ii) $0.4-1.5 \mathrm{ps}$ (the high energy state).

\section{A. Excitation of the ILM}

Figure 3 displays the change in the distribution of total energy of an atom, $E_{i}$, in the $(5,5)$ CNT during $0.1-0.4 \mathrm{ps.}$ There exists a pair of carbon atoms that maintained a considerably high energy state for $300 \mathrm{fs}$. Those are denoted as atoms A and B in the white circle in Fig. 3. Such energy concentration for several hundred femtoseconds is one of the characteristics of the ILMs in the atomic scale. In addition, the previous study ${ }^{14}$ revealed the vibration mode of the ILM in a graphite sheet in which two neighboring atoms strongly oscillate along their bonding direction. This agrees well with the behavior of atoms A and B in the $(5,5)$ CNT.

Here, the frequency of atoms A and B is examined and compared with the maximum in the phonon-dispersion relation because the vibrational frequency of the ILMs exceeds the upper bound of the phonon bands. Figure 4 shows the power spectrum distribution of atoms A and B indicated in Fig. 3. For comparison, the upper bound of the phonon bands in the $(5,5) \mathrm{CNT}$ at the strain of $\varepsilon_{z z}=0.10, \omega_{\max }^{\text {phonon }}$, is also shown as a dashed vertical line. The spectrum was calculated
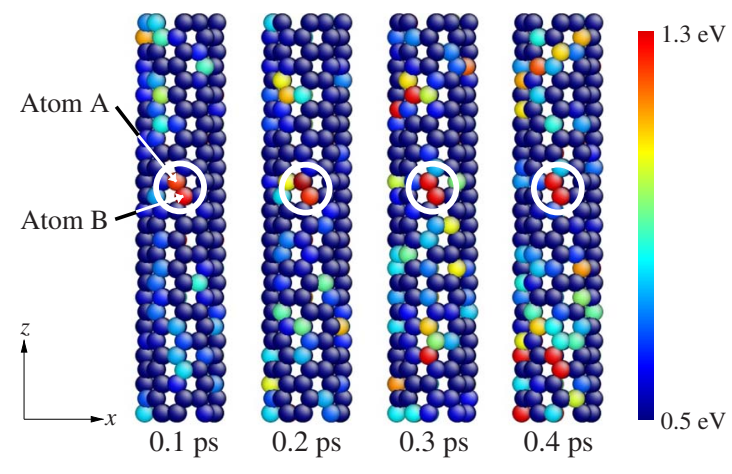

FIG. 3. (Color online) Change in distribution of the total energy of an atom, $E_{i}$, in the $(5,5) \mathrm{CNT}$ under axial tension during $0.1-0.4$ ps. 

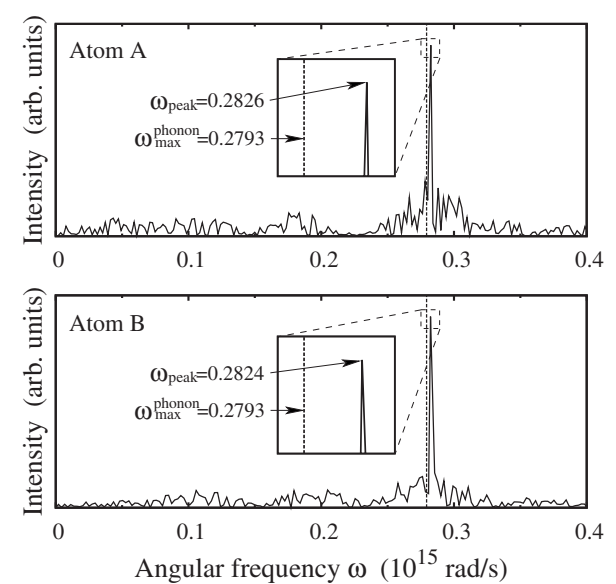

FIG. 4. Power spectrum distribution of atoms A and B (see Fig. 3 ) in the top and bottom panels, respectively. The dominant (peak) frequency is indicated as $\omega_{\text {peak }}$. For comparison, the upper bound of the phonon bands, $\omega_{\max }^{\text {phonon }}$, is also shown as a vertical dashed line.

by the discrete Fourier transform of the atomic displacementtime relation during $0.1-0.4 \mathrm{ps}$ with a sampling interval of 0.1 fs. The detailed procedure was described in a previous report. ${ }^{14}$ Here, the atomic displacement was taken along the vibration direction because this is the dominant moving orientation of the atom according to the trajectory of the velocity vector. The dominant frequency of atoms $\mathrm{A}$ and $\mathrm{B}$, $\omega_{\text {peak }}=0.2826 \times 10^{15}$ and $0.2824 \times 10^{15} \mathrm{rad} / \mathrm{s}$, respectively, exceeded the maximum phonon frequency, $\omega_{\max }^{\text {phon }}=0.2793$ $\times 10^{15} \mathrm{rad} / \mathrm{s}$. This evidently manifested the ILM excitation at the atoms A and B. The ILM vibration stably continued for $300 \mathrm{fs}$, which corresponds to about 14 cycles. The detailed excitation process and characteristics of the ILM is almost same as those obtained in the previous studies. ${ }^{16}$

In this study, we initially imposed a somewhat special condition of atomic displacement on the armchair CNT, with the aim of exciting the ILMs, but this condition was never unphysical but actually exists because the displacement corresponds to one of the normal vibrational modes of the phonons. In addition, the conditions required for the ILM excitation are expected to be satisfied by thermal fluctuations, considering the minute time scale for atomic-level phenomena (femtosecond or picosecond order) with respect to a second order. Thus, we expect the ILM to be excited in real CNTs.

\section{B. Stone-Wales transformation triggered by ILM}

Figure 5 shows the change in atomic configuration and distribution of total energy of an atom, $E_{i}$, during $0.4-1.5$ ps. A remarkable structural change first appeared during $0.4-0.5$ ps, as the $\mathrm{C}-\mathrm{C}$ bond between atoms $\mathrm{A}$ and $\mathrm{B}$ along the ILM vibration direction broke [Figs. 5(a) and 5(b)]. This initial breaking of the bond leads to a considerable increase in the maximum energy of an atom, $E_{\max }$ (see also Fig. 2), which is a clear sign of the initiation of mechanical instability. Subsequently, the second neighboring $\mathrm{C}-\mathrm{C}$ bond broke at $1.2 \mathrm{ps}$ [Fig. 5(c)] while the first rebonding occurred with the formation of a pentagon and heptagon at 1.3 ps [Fig. 5(d)]. Finally,
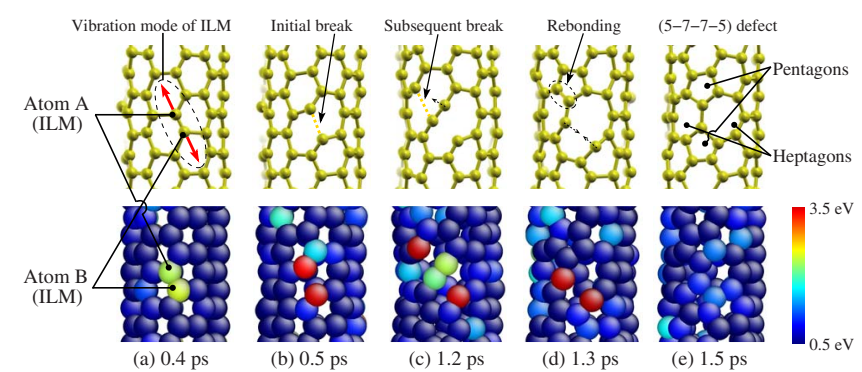

FIG. 5. (Color online) Change in atomic configuration (top) and distribution of total energy of an atom, $E_{i}$, (bottom) during $0.4-1.5$ ps. Only the vicinity of the ILM-excited atoms A and B is shown for clarity. The red (gray) arrows at 0.4 ps indicate the vibration mode of ILM.

the (5-7-7-5) defect consisting of a double pentagonheptagon was formed at $1.5 \mathrm{ps}$ by the second rebonding at the lower site [Fig. 5(e)]. At the same time, $E_{\max }$ dropped to a lower energy level, suggesting that the atomic structure reached a new stable configuration. The breaking and subsequent rebonding of the $\mathrm{C}-\mathrm{C}$ bonds obtained here is the typical process of the Stone-Wales transformation that was confirmed by $a b$ initio molecular-dynamics simulations. ${ }^{20}$

It should be noted that we carried out another MD simulation under the constant energy only applying an initial momentum corresponding to $1400 \mathrm{~K}$, which was the averaged temperature during the ILM excitation, but we found neither a Stone-Wales transformation nor an ILM, even for a longer simulation period of 100 ps. This implies that the StoneWales transformation observed in this study was not induced by the normal thermal fluctuations but by the ILM. In fact, the Stone-Wales transformation was observed at a higher temperature, 2000-3000 K, under the same or higher strain conditions. ${ }^{19,20}$

Figure 6 shows the change in displacement along the vibration direction of atoms A and B through the ILM excitation and initial breaking of a $\mathrm{C}-\mathrm{C}$ bond. The vibrational amplitude of atoms A and B gradually increased with slight fluctuation as time passed. This increase in amplitude was also characteristically observed just before and during the ILM excitation in a graphite sheet because the nonlinearity in (a) Atom A

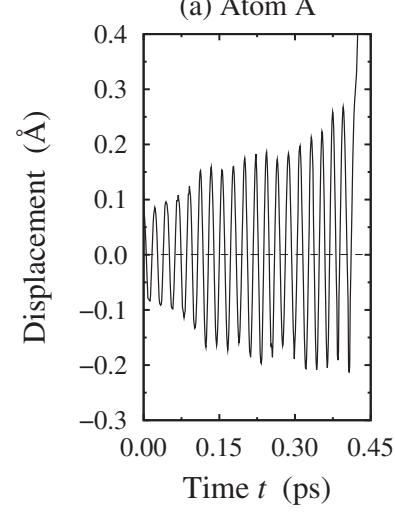

(b) Atom B

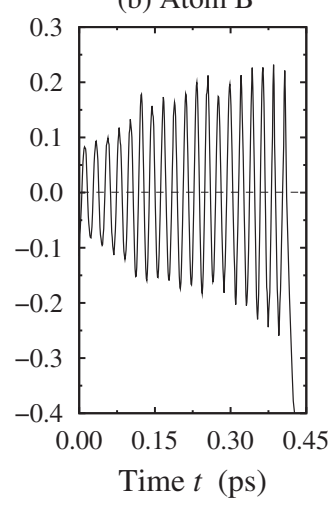

FIG. 6. Change in displacement of atoms A and B in the left and right panels, respectively. The atomic displacement is taken along the vibration axis of the ILM. 
the $\mathrm{C}-\mathrm{C}$ interaction that predominates the excitation and fundamental properties of ILMs ${ }^{15,16}$ strongly enhances the localized vibration. ${ }^{14}$ In fact, the ILM cannot be excited in CNTs with a weak nonlinearity while the stronger nonlinearity excites the ILM with a higher frequency and longer lifetime. ${ }^{16}$ After the amplification of the ILM vibration during 0.1-0.4 ps, the displacement of atoms $\mathrm{A}$ and $\mathrm{B}$ increased dramatically, which corresponds to the initial breaking of their own $\mathrm{C}-\mathrm{C}$ bond in the Stone-Wales transformation. This suggests that the bond breaking arose from the intensive energy concentration exceeding the activation barrier due to the amplified ILM vibration. In other words, the highly amplified ILM triggered the Stone-Wales transformation in the $(5,5) \mathrm{CNT}$ under tension.

Since the Stone-Wales transformation is one of the mechanical instabilities in the atomic scale, the results obtained in this study suggest that such mechanism that a local bond switching in a homogeneous atomic structure can be triggered by the ILM could be potentially applied to other mechanical instabilities, e.g., as an origin of phase transformations in silicon under hydrostatic pressure or multiaxial conditions. ${ }^{27}$ The present authors expect that the atomic-level study of the ILMs connected to mechanical instability will be further developed and this work will help pave the way to understanding some of the variety of complex mechanical instability issues.

\section{CONCLUSION}

MD simulations were conducted for a $(5,5)$ armchair CNT under axial tension with the aim to elucidate the role of ILMs in the atomic scale, as a possible trigger of the Stone-Wales transformation that locally produces a topological defect consisting of two pentagons and two heptagons coupled in pairs (5-7-7-5). In the early stage of the MD simulation with an initial displacement of the zone-boundary phonon mode and a minute fluctuation, a strong vibration of a pair of neighboring atoms was observed at a local site of the CNT, where kinetic energy concentrated and this state continued for several hundred femtoseconds. The discrete Fourier transformation revealed that the frequency of the localized vibration exceeded the upper bound of the phonon bands. This evidently indicates that the ILM was successfully excited from the modulational instability of the zone-boundary phonon mode.

The excited ILM was gradually amplified by the nonlinearity of C-C interaction, which always plays a central role in determining the excitation and fundamental properties of the ILMs. The amplified ILM vibration, finally, induced a $\mathrm{C}-\mathrm{C}$ bond breaking at the excitation site that leads to the Stone-Wales transformation because the intense energy concentration by the ILM exceeded the activation barrier. In other words, the highly amplified ILM triggered the mechanical instability of the Stone-Wales transformation in the $(5,5)$ CNT under axial tension.

This atomic-level mechanism, ILM-derived mechanical instability, can be universally applicable to other situations. For example, it may be an origin of phase transformation in silicon under hydrostatic pressure or multiaxial stress. Further study of the connection between the ILMs and the mechanical instability will be performed in the near future.

\section{ACKNOWLEDGMENT}

This work was supported in part by a Grant-in-Aid for Challenging Exploratory Research (Grant No. 21656031) of the Japan Society of the Promotion of Science.

\footnotetext{
*shimada@cyber.kues.kyoto-u.ac.jp

${ }^{1}$ A. J. Sievers and S. Takeno, Phys. Rev. Lett. 61, 970 (1988).

${ }^{2}$ S. Takeno, K. Kisoda, and A. Sievers, Prog. Theor. Phys. Suppl. 94, 242 (1988).

${ }^{3}$ S. R. Bickham, S. A. Kiselev, and A. J. Sievers, Phys. Rev. B 47, 14206 (1993).

${ }^{4}$ S. A. Kiselev, S. R. Bickham, and A. J. Sievers, Phys. Rev. B 50, 9135 (1994).

${ }^{5}$ B. F. Feng, Y. Doi, and T. Kawahara, Physica D 214, 33 (2006).

${ }^{6}$ Y. Doi, Phys. Rev. E 68, 066608 (2003).

${ }^{7}$ J. Cuevas, C. Katerji, J. F. R. Archilla, J. C. Eilbeck, and F. M. Russell, Phys. Lett. A 315, 364 (2003).

${ }^{8}$ J. L. Marín, J. C. Eilbeck, and F. M. Russell, Phys. Lett. A 248, 225 (1998).

${ }^{9}$ E. Trías, J. J. Mazo, and T. P. Orlando, Phys. Rev. Lett. 84, 741 (2000).

${ }^{10}$ P. Binder, D. Abraimov, A. V. Ustinov, S. Flach, and Y. Zolotaryuk, Phys. Rev. Lett. 84, 745 (2000).

${ }^{11}$ H. S. Eisenberg, Y. Silberberg, R. Morandotti, A. R. Boyd, and J. S. Aitchison, Phys. Rev. Lett. 81, 3383 (1998).

${ }^{12}$ M. Sato, B. E. Hubbard, A. J. Sievers, B. Ilic, D. A. Czaplewski, and H. G. Craighead, Phys. Rev. Lett. 90, 044102 (2003).

${ }^{13}$ M. Sato, B. E. Hubbard, A. J. Sievers, B. Ilic, and H. G. Craighead, Europhys. Lett. 66, 318 (2004).
}

${ }^{14}$ Y. Yamayose, Y. Kinoshita, Y. Doi, A. Nakatani, and T. Kitamura, EPL 80, 40008 (2007).

${ }^{15}$ Y. Kinoshita, Y. Yamayose, Y. Doi, A. Nakatani, and T. Kitamura, Phys. Rev. B 77, 024307 (2008).

${ }^{16}$ T. Shimada, D. Shirasaki, Y. Kinoshita, Y. Doi, A. Nakatani, and T. Kitamura (unpublished).

${ }^{17}$ T. Shimada, S. Okawa, S. Minami, and T. Kitamura, Mater. Sci. Eng., A 513-514, 166 (2009).

${ }^{18}$ A. J. Stone and D. J. Wales, Chem. Phys. Lett. 128, 501 (1986).

${ }^{19}$ M. Buongiorno Nardelli, B. I. Yakobson, and J. Bernholc, Phys. Rev. Lett. 81, 4656 (1998).

${ }^{20}$ M. Buongiorno Nardelli, B. I. Yakobson, and J. Bernholc, Phys. Rev. B 57, R4277 (1998).

${ }^{21}$ J. Tersoff, Phys. Rev. Lett. 61, 2879 (1988).

${ }^{22}$ J. Tersoff, Phys. Rev. B 37, 6991 (1988).

${ }^{23}$ D. W. Brenner, Phys. Rev. B 42, 9458 (1990).

${ }^{24}$ E. Bitzek, P. Koskinen, F. Gähler, M. Moseler, and P. Gumbsch, Phys. Rev. Lett. 97, 170201 (2006).

${ }^{25}$ T. Cretegny, T. Dauxois, S. Ruffo, and A. Torcini, Physica D 121, 109 (1998).

${ }^{26}$ L. Verlet, Phys. Rev. 159, 98 (1967).

${ }^{27}$ S. M. Jeong and T. Kitamura, Jpn. J. Appl. Phys., Part 1 46, 5924 (2007). 